\title{
Post-operative Post Irradiation using Ultrasound Breast Tumor
}

\author{
Dalia Aly Mohamed Aly Abou Taleb* \\ Demonstrator, Faculty of Applied Medical Sciences, Misr University for Science and Technology, Egypt
}

Submission: November 29, 2019; Published: December 17, 2019

"Correspondence Author: Dalia Aly Mohamed Aly Abou Taleb, Demonstrator, Faculty of Applied Medical Sciences, Misr University for Science and Technology, Egypt

\section{Editorial}

\section{Post-operative Breast}

Treatment to the breast by surgery or radiotherapy produces a number of changes. These alterations change with time, and it can take at least 6-18 month before the tissue changes are stable. Local excision is more frequent and so the importance of postoperative ultrasound breast examination is increasing.

\section{Post-Surgery}

The commonest abnormality is a hematoma, producing a rounded lesion which, in the early stages is, ill defined but which later becomes better defined. Blood clots adjacent to the inner wall of the haematoma may mimic a solid lesion. Associated to this there may be skin thickening, focally increased reflectivity and trabecular distortion, producing quite marked focal attenuation. Eventually a scar results, seen sonographically as a narrow band of attenuation extending down from the skin deeper in the tissue, perhaps persisting trabecular distortion and skin thickening.

\section{Post Irradiation}

After irradiation the most common changes are skin thickening, increased reflectivity of the subcutaneous fat, and thickening of cooper's ligaments and architectural distortion of the tissue. Thickening of the skin (normally less than $3 \mathrm{~mm}$ ) is present in $60-95 \%$ of patients and is best evaluated in comparison to the opposite breast. The thickened skin can be poorly reflective. The increased reflectivity is also characteristic and results in a decreased contrast between the fat and the glandular tissue. The image is similar to lymphoedema ans is probably due to increased interstitial fluid. In the early post irradiation phase, fluid sometimes accumulates between the fat lobules. These changes gradually improve over the first year, but in most cases some alterations persist. Ultrasound of the irradiated breast is indicated to detect tumor recurrence.
To summarize the role of breast ultrasound we can conclude that; ultrasound is one of the most popular modalities and has many advantages and limitations.

- $\quad$ Advantages include that:

i. Ultrasound is very useful and widely accepted as an adjunct to mammography, the sensitivity for carcinoma has been recorded by several authors at up to $92-95 \%$.

ii. Ultrasound is highly reliable in differentiating benign from malignant lesions, for palpable as well as impalpable masses.

iii. If ultrasound is used in conjunction withX-ray mammography, the sensitivity for the pre - operative identification of cancer rises to $97 \%$

iv. Follow up of postoperative scar tissue.

v. Ultrasound is capable of reducing benign - malignant biopsy ratio , and ratio of 1:1 or lower should be a goal.

vi. Ultrasound guided biopsy can make surgery for benign lesions obsolete and favors a one -stage operation in malignant lesions.

\section{Limitations}

a. Sonography is actually not well suited to screening for occult breast cancer because the detection of asymptomatic carcinoma not detected on mammography, is limited, and the examination is highly operator dependent and time - consuming, making it economically difficult to justify.

b. Detection of micro calcifications, an important landmark for early cancer on mammography, is limited on ultrasound. Although the detection of micro calcifications is improved with modern equipment (up to $100 \%$ when inside a mass , using 10 
MHZ probes) , dispersed calcifications in the tissue are less well depicted.

c. Large breasts with fatty involution are difficult to examine, and small deeply located masses maybe overlooked with sonography.

d. Sonography is not routinely a first - choice examination except for some particular situations as in pregnant or lactating patients, to avoid irradiation and because of the mammographically dense breast.

\section{Three - Dimentional Ultrasound}

Two -dimentional ultrasound imaging of female breast is useful in determining if a mass is cystic or solid but is less useful for cancer screening. Recent work suggests that three -dimensional ultrasound 3DUS, alone or in combination with color/pulsed dopller, and contrast agents, may be helpful in differentiating benign from malignant lesions, including both surface features and internal architecture. Arbitrary plane evaluation of a breast volume permits planes parallel to chest wall to be evaluated, providing a more complete evaluation than two - dimensional ultrasound. Although two- dimensional ultrasound 2DUS has been shown to be valuable for differentiating some aspects of benign/malignant disease. Three-dimensional ultrasound (3DUS) imaging of breast may assist this area significantly. 3DUS improved evaluation of focal infiltrates, intracystic structure and multifocal disease plus re-evaluation of data after the patient has left the clinic.

3DUS also can be useful for assisting needle localization and guidance for biopsy purposes . 3DUS imaging of the breast should allow for direct visualization of sonographically detectable mass lesions, resulting in better analysis of those lesions both with grayscale and color doppler flow imaging using both arbitrary planer review and volume - rendering techniques. It is important to first identify the parameters used to evaluate breast masses with 2DUS:

i. Internal architecture

ii. Shape

iii. Boundary echo margins

iv. Direction of the long axis

\section{Advantages of 3DUS}

a. Differentiate benign from malignant masses with better judgement on the evidence of focal infiltration of tumor

b. Arbitrary planer review permits through evaluation of masses

c. Plans parallel to chest wall can be evaluated

d. Surface features of masses can be evaluated

e. Vascularity of masses can be evaluated in 3D 